\title{
Influencia del extendido de cola en la resistencia de tableros contrachapados de lupuna (Ceiba insignes (Kunth) P.E. Gibbs \& Semir)
}

\author{
Influence of adhesive spread on the strength of plywood boards of lupuna \\ (Ceiba insignes (Kunth) P.E. Gibbs \& Semir)
}

Rosa M. Loza de la Cruz ${ }^{1}$, Leónidas Miguel Castro² y Julio C. Canchucaja Rojas²

\begin{abstract}
Resumen
En el presente trabajo se evalúo la influencia de cinco niveles de extendido de cola en la resistencia a la línea de cola en la elaboración de tableros contrachapados de "lupuna" (Ceiba insignes (Kunth) P.E. Gibbs \& Semir) de tres capas. Los resultados obtenidos demuestran que la resistencia a la tracción paralela al grano de las muestras varía en promedio desde $15,88 \mathrm{~kg} / \mathrm{cm}^{2}$, para un extendido de $100 \mathrm{~g} / \mathrm{m}^{2}$, hasta $19,84 \mathrm{~kg} / \mathrm{cm}^{2}$ que corresponde a un extendido de $250 \mathrm{~g} / \mathrm{m}^{2}$. Asimismo, los mejores valores de resistencia a la humedad se encuentra en el rango de extendido de 250 a $300 \mathrm{~g} / \mathrm{m}^{2}$.
\end{abstract}

Palabras clave: extendido de cola; lupuna; Ceiba insignes; tableros contrachapados.

\begin{abstract}
In this article, we evaluated the influence of five levels of extended glue in the resistance to the line of glue in the production of plywoods of "lupuna" (Ceiba insignes (Kunth) P.E. Gibbs \& Semir) of three layers. The results showed that the tensile strength parallel to grain of the samples varies in average from $15,88 \mathrm{~kg} / \mathrm{cm}^{2}$, for a spread of $100 \mathrm{~g} / \mathrm{m}^{2}$, until $19,84 \mathrm{~kg} / \mathrm{cm}^{2}$ which corresponds of an extended to a $250 \mathrm{~g} / \mathrm{m}^{2}$. Moreover the best values of resistance to humidity are found in the range extended from 250 to $300 \mathrm{~g} / \mathrm{m}^{2}$.
\end{abstract}

Key words: extended glue; lupuna; Ceiba insignes; plywood.

\footnotetext{
${ }^{1}$ Servicio Nacional Forestal y de Fauna Silvestre (SERFOR), Perú. E-mail: rloza@serfor.gob.pe

${ }^{2}$ Facultad de Ciencias Forestales, Universidad Nacional Agraria La Molina, Perú.
} 


\section{Introducción}

El libre comercio y la globalización de los mercados exigen actualmente productos de calidad y bajos costos, por lo que las industrias tienen que utilizar con mayor eficiencia la materia prima, la tecnología y la mano de obra para obtener un producto competitivo en el mercado nacional e internacional.

En este contexto, la industria de tableros contrachapados en la actualidad tiene que competir en calidad con sus similares importados; esta situación ha mostrado el estancamiento tecnológico en que se encuentra, teniendo que dirigir su producción básicamente al mercado local. La falta de una modernización en su maquinaria y equipo, utilización de nuevas especies, falta de personal capacitado, carencia de investigación, entre otros factores, han permitido esta situación.

De otro lado, en la estructura de costos, la resina constituye aproximadamente el $40 \%$ del costo del tablero contrachapado, por lo que se debe poner especial interés, tanto en la utilización de la resina, que es el componente principal de la cola, como su correcta aplicación en la conformación de los tableros.

El extendido se define como la cantidad de cola esparcida sobre el área de la lámina a encolar, se expresa en gramos sobre metros cuadrados $\left(\mathrm{g} / \mathrm{m}^{2}\right)$. Heritage (1983) conceptualiza a la adhesión, como el estado en el cual dos superficies son mantenidas juntas mediante la acción de fuerzas interfaciales, las cuales pueden consistir de fuerzas de valencia, acción interbloqueantes o ambas. Estas fuerzas de atracción de las superficies se deben a las fuertes interacciones de las moléculas, átomos e iones en ellas, donde al activarse desembocan en una adhesión fuerte.

Heritage (1983), Kollmann (1984) y Poblete (2001) indican que, en la adhesión se pueden reconocer tres tipos de fuerza que mantienen la unión la cohesión, que corresponde a la fuerza desarrollada entre las moléculas de adhesivo; este tipo se forma sólo en la línea de cola. Estas fuerzas influyen directamente sobre la resisten- cia de la línea de cola e indirectamente sobre la adhesión mecánica. Así con mejor cohesión se tendrá dedos de cola más resistente, la adhesión específica, fuerza de naturaleza química desarrollada entre moléculas del adhesivo y la superficie de madera y la adhesión mecánica, que corresponde al anclaje mecánico provocado por la penetración del adhesivo en la madera.

Heritage (1983) indica que las láminas antes de ser encoladas deben tener un espesor uniforme, una superficie lisa, plana y un contenido de humedad dentro del rango según el tipo de adhesivo a utilizar. Al respecto, BASF (1981) manifiesta que las láminas encoladas con resinas a base de urea deben tener un contenido de humedad de 8 a $10 \%$.

Watkins (1980) menciona que la única razón por la que falla una cola de unión perfecta podría ser una chapa de mala calidad, cola mal extendida o mala condición de prensado. Este autor sostiene que la forma de evaluar la calidad de los tableros está sobre la base de la falla de la madera; si el área rota es toda la madera o cubre con fibras de madera completamente, se tendrá en ese caso una falla en la madera del 100\%. Respecto al extendido, BASF (1981) recomienda aplicar una cantidad de 120 - 180 $\mathrm{g} / \mathrm{m}^{2}$. VENCEDOR (1989) sugiere un extendido de $200-240 \mathrm{~g} / \mathrm{m}^{2}$. Asimismo en las investigaciones sobre tableros contrachapados realizadas en la Universidad Nacional Agraria La Molina por Córdova (1992), Paredes (1995), Duda (1996) y Canchucaja (2001), en los cuales utilizaron extendidos de $220 \mathrm{~g} / \mathrm{m}^{2}$ con excepción de Canchucaja (2001) que utilizó 240 g/ $\mathrm{m}^{2}$; obtuvieron valores promedio de resistencia a la tracción de 10,$6 ; 15,8 ; 15,4$ y $14,1 \mathrm{~kg} / \mathrm{cm}^{2}$, respectivamente.

En la presente investigación se evalúo la influencia de cinco niveles de extendido de cola en la resistencia a la línea de cola de tableros contrachapados de "lupuna" (Ceiba insignes (Kunth) P.E. Gibbs \& Semir) de tres capas. Estos resultados permitirán elegir el extendido de cola óptimo y reducir los costos de fabricación del producto sin mermar la calidad mecánica de los tableros. 


\section{Materiales y Métodos}

El presente trabajo se realizó en los Laboratorios de Fabricación de Paneles, Ensayos Tecnológicos, Trabajabilidad y Transformación Química de la Madera del Departamento de Industrias Forestales de la Universidad Nacional Agraria La Molina (Lima, Perú).

Se utilizaron láminas de lupuna (Ceiba insignes (Kunth) P.E. Gibbs \& Semir), de 1,3 mm de espesor, las cuales fueron adquiridas de la empresa General Products Co SA.

La mezcla encolante se preparó utilizando los siguientes porcentajes de aditivos en la formulación, urea formaldehído (53,5\%), harina de trigo $(26,7 \%)$, agua $(18,7 \%)$ y como catalizador cloruro de amonio $(1,1 \%)$.

Se elaboraron tableros de tres capas de $60 \mathrm{x}$ $60 \times 3,8 \mathrm{~mm}$. Para el encolado de los tableros se utilizaron extendidos de 100, 150, 200, 250 y $300 \mathrm{~g} / \mathrm{m}^{2}$, con cinco repeticiones por cada tratamiento.

La aplicación de la cola se realizó en forma manual y se preparó un $10 \%$ adicional para cada tablero, a fin de prever las posibles pérdidas durante el proceso de encolado.

Los tableros fueron prensados en una prensa de platos calientes a una temperatura de $125^{\circ} \mathrm{C}$, una presión específica de $6 \mathrm{~kg} / \mathrm{cm}^{2}$ por un tiempo de 2 minutos.

Para evaluar la resistencia de la línea de cola a los esfuerzos de tensión, se procedió de acuerdo a la Norma ASTM D 906 - 64 (ASTM 1976). Se prepararon probetas de $2,54 \mathrm{~cm} \mathrm{x}$ $8,26 \mathrm{~cm}$, para los ensayos de resistencia paralela al grano.
Para evaluar la resistencia de los tableros a la humedad, se procedió de acuerdo a lo indicado en la Norma Técnica Peruana ITINTEC 251.042. Este ensayo consiste en una inmersión de las probetas en agua, a temperatura ambiente $\left(20^{\circ} \mathrm{C} \pm 5\right)$ durante 24 horas. Con la ayuda de un punzón se trata de separar las láminas de maderas por la línea de cola. Si se observa que las láminas se separan completamente por la línea de cola sin desgarrar la madera tendrá un puntaje de 0 , mientras que si la lámina se separa desgarrando la madera sin abrirse la línea de cola, se dará un puntaje de 10 .

Para evaluar estadísticamente los resultados de la resistencia a la línea de cola a la tracción paralela al grano, se utilizó un Diseño Completamente al Azar (DCA) y la prueba de Tukey entre tratamientos.

Para evaluar estadísticamente la influencia de la resistencia a la humedad se realizó la prueba de comparación no paramétrica de Kruskall - Wallis, que efectúa una prueba de comparación de medianas entre tratamientos.

\section{Resultados}

Los resultados del ensayo de resistencia a la tracción paralela al grano se muestran en el Cuadro 1 y en la Figura 1. Se observa que al utilizar diferentes niveles de extendido, los valores de la resistencia a la tracción paralela al grano de las muestras varían, en promedio, desde $15,88 \mathrm{~kg} / \mathrm{cm}^{2}$ para un extendido de 100 $\mathrm{g} / \mathrm{m}^{2}$, hasta $19,84 \mathrm{~kg} / \mathrm{cm}^{2}$ que corresponde a un extendido de $250 \mathrm{~g} / \mathrm{m}^{2}$. La prueba de comparación múltiple por pares de tratamiento (niveles de extendido de cola) de Tukey, muestra que

\begin{tabular}{|c|c|c|c|c|c|c|c|}
\hline \multirow{2}{*}{ Tratamiento } & \multirow{2}{*}{$\begin{array}{c}\text { Extendido de } \\
\mathbf{c o l a}\left(\mathbf{g} / \mathbf{m}^{\mathbf{2}}\right)\end{array}$} & \multicolumn{5}{|c|}{ Promedio de resistencia del tablero $\left(\mathbf{k g} / \mathbf{c m}^{\mathbf{2}}\right)$} & \multirow{2}{*}{ Promedio } \\
& & Tablero 1 & Tablero 2 & Tablero 3 & Tablero 4 & Tablero 5 & (kg/cm² \\
\hline 1 & 100 & 14,53 & 13,73 & 16,18 & 17,53 & 17,42 & 15,88 \\
\hline 2 & 150 & 16,18 & 17,14 & 17,25 & 18,34 & 18,19 & 17,42 \\
\hline 3 & 200 & 18,24 & 19,03 & 18,87 & 18,74 & 18,06 & 18,59 \\
\hline 4 & 250 & 19,64 & 23,08 & 18,98 & 19,11 & 18,39 & 19,84 \\
\hline 5 & 300 & 16,25 & 17,95 & 18,10 & 19,01 & 16,59 & 17,58 \\
\hline
\end{tabular}

Cuadro 1. Valores de resistencia a la tracción paralela al grano de los tableros elaborados con diferentes niveles de extendido de cola. 


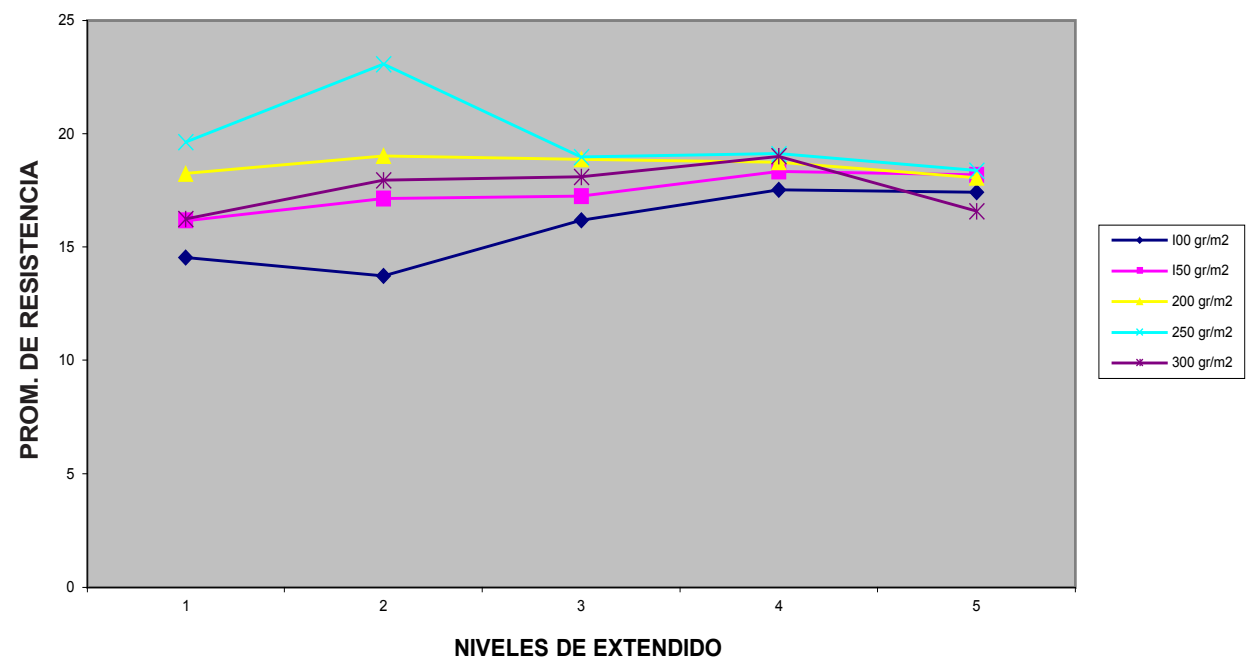

Figura 1. Resultados del ensayo de resistencia a la línea de cola con diferentes niveles de extendido de cola.

existe diferencias significativas de resistencias promedio a la tracción paralela al grano entre los niveles de 100 y $200 \mathrm{~g} / \mathrm{m}^{2}$, y entre los niveles de extendido 100 y $250 \mathrm{~g} / \mathrm{m}^{2}$.

En el Cuadro 2 se muestran los resultados de la resistencia a la humedad de los tableros fabricados con distintos niveles de extendidos de cola. Las cifras indican una relación directamente proporcional entre estas variables; es decir a mayor extendido de cola se obtiene una mayor resistencia promedio a la humedad. Asimismo, al aplicar la prueba no paramétrica de Kruskal - Wallis a los resultados obtenidos se encontró que al menos uno de los tratamientos de la resistencia a la humedad es diferente a los demás y que el mayor puntaje corresponde a un extendido de $300 \mathrm{~g} / \mathrm{m}^{2}$.

\section{Discusión}

Los resultados promedio de resistencia a la tracción paralela al grano guardan relación con los valores de extendido utilizados en las investigaciones realizadas por Córdova (1992), Paredes (1995), Duda (1996) y Canchucaja (2001). También se aprecia que al aumentar el nivel de extendido desde $100 \mathrm{~g} / \mathrm{m}^{2}$ hasta $250 \mathrm{~g} /$ $\mathrm{m}^{2}$, la resistencia a la tracción paralela al grano va en aumento; mientras que cuando se pasa a un nivel de extendido de $250 \mathrm{~g} / \mathrm{m}^{2}$ a $300 \mathrm{~g} /$ $\mathrm{m}^{2}$ la resistencia a la tracción paralela al grano disminuye.

Esto podría deberse a las características intrínsecas que explican la teoría de adhesión, según Poblete (2001), la adhesión es obtenida

\begin{tabular}{|c|c|c|c|c|c|c|c|}
\hline \multirow{2}{*}{ Tratamiento } & \multirow{2}{*}{$\begin{array}{c}\text { Extendido de } \\
\text { cola (g/m }\end{array}$} & \multicolumn{5}{|c|}{ Resistencia a la humedad } & \multirow{2}{*}{ Promedio } \\
\cline { 3 - 7 } & Tablero 1 & Tablero 2 & Tablero 3 & Tablero 4 & Tablero 5 & \\
\hline 1 & 100 & 4 & 6 & 4 & 5 & 4 & 4,60 \\
\hline 2 & 150 & 6 & 6 & 6 & 6 & 6 & 6,00 \\
\hline 3 & 200 & 6 & 6 & 6 & 6 & 6 & 6,00 \\
\hline 4 & 250 & 6 & 8 & 7 & 6 & 8 & 7,00 \\
\hline 5 & 300 & 8 & 7 & 8 & 8 & 8 & 7,80 \\
\hline
\end{tabular}

Cuadro 2. Resultados del ensayo de resistencia a la humedad de los tableros elaborados con diferentes niveles de extendido de cola. 
en un $95 \%$ por la naturaleza de las uniones químicas que se hayan formado durante el fraguado, tales como valencia, covalencia y puentes de hidrógeno. Lo cual significa que existe un balance óptimo, entre las características de la madera y del adhesivo para que se presente un buen fraguado de cola. Para este ensayo los valores óptimos de extendido de cola, según los valores de resistencia paralela al grano, se encuentran entre 200 y $250 \mathrm{~g} / \mathrm{m}^{2}$.

Los mayores valores de resistencia a la humedad corresponden a los extendidos de cola de 250 a $300 \mathrm{~g} / \mathrm{m}^{2}$, estos resultados se podrían considerar como aceptables considerando que inclusive son superiores a los obtenidos por Córdova (1992), Paredes (1995), Duda (1996) y Canchucaja (2001); teniendo en cuenta además que la urea formaldehído, según Chugg (1964), no tiene necesariamente una elevada resistencia a la humedad. Se debe considerar que los ensayos de resistencia a la humedad se consideran técnicamente apropiados usando un extendido de $250 \mathrm{~g} / \mathrm{m}^{2}$ por economía industrial.

\section{Conclusiones}

La mayor resistencia a la tracción paralela al grano se encuentra en un rango de 200 a 250 g/ $\mathrm{m}^{2}$ de extendido de cola.

La mayor resistencia a la humedad se encuentra en el rango de 250 a $300 \mathrm{~g} / \mathrm{m}^{2}$ de extendido de cola.

Los valores de resistencia a la tracción paralela al grano se incrementa hasta un extendido de $250 \mathrm{~g} / \mathrm{m}^{2}$, por encima de este valor dicha resistencia disminuye.

\section{Bibliografía}

ASTM (1976). Standard Test Method for Hydrogen Ion Concentration of Dry Adhesive Filme. 2p.

BASF, Peruana S.A. 1981. Resinas Sintéticas Kaurit 390. Folleto Técnico. Lima. PE. 4p.

Blomquist, R. 1981. Educational modules for materials science and engineering. Vol. III. Pennsylvania, US.120p.
Canchucaja, J. 2001. Influencia de la presión especifica en el prensado de tableros contrachapados de lupuna (Chorisia integrifolia). Tesis Ing. Forestal. UNALM. PE. 70p.

Chugg, W. 1964. The theory and practice of glued laminated timber structures. London. Ernest Benn Limited. GB. 423p.

Córdova, R. 1992. Harina de madera como carga en la formulación de tableros contrachapado. Tesis de Mg. Sc. UNALM. PE. 92p.

Duda, O. 1996. Uso de la harina de yuca en la formulación de cola para tableros contrachapados. Tesis Ing. Forestal. UNALM. PE. 96p.

Heritage, C. 1983. Adhesive bonding of wood and other structural material. Pensilvania. Vol III. Ed. Blomquiest. US. 450p.

Kollman, F. 1984. Principles of wood science and technology. Vol. 1. Berlin. DE. 592p.

Mullins, JE; McKnight, ST. 1981. Canadian woods: their properties and uses. Otawa, CN. 389p.

Paredes, A. 1995. Harina de plátano como extendedor en la formulación de cola para tableros contrachapados. Tesis Ing. Forestal. UNALM. PE. 144p.

Poblete, H. 2001. Tablero de partículas. Facultad de Ciencias Forestales. Universidad Austral de Chile. CL. 177p.

VENCEDOR. 1989. Vence UF - 600: catálogo de resinas a base de urea formaldehído. Lima. PE. 5p.

Watkins, E. 1980. Principles of plywood productions. California. US. 39p. 